\title{
Evaluación semicuantitativa del flujo cerebral con Doppler-duplex en recién nacidos de término con encefalopatía hipóxico-isquémica
}

\author{
Manuel Fernández A. ${ }^{1}$; M. Angélica Oto $\mathrm{L}^{2}$; Karla Moënne $\mathrm{B} .^{{ }^{1} \text {; }}$ \\ Carlos Acevedo Sch. ${ }^{3} ;$ Jaime Burgos M. $^{4}$ \\ Doppler-duplex Pourcelot's index in neonates \\ with and without hypoxic-ischemic encephalopathy
}

\begin{abstract}
Doppler-duplex (O-D) can be used to measure blood flow. In the brain, semi-quantitative measurement can be done using Pourcelot index (PI). PI usually falts 24 or more hours after the period of asphyxia because of reactive hyperaemia which increases cerebral blood flow to above pre-ischemic levels. A control group of 25 healthy terms infants were studied with D.D. Also 42 term infants with hypoxic-ischern ic encephalopsthy (HIE). who survived for more than 8 days were consecutively studled. Twenty-two were mild HIE, 16 moderate and 4 severe. $D$-D was done at dav 4 in control group and at day 5.6 and 10 in mild, moderate and severe HIE respectively. Average PI was $0.680 \pm 0.038$ in control group and $0.668 \pm 0.058,0.637 \pm 0.081$ and $0.677 \pm 0.085$ in mild, moderate and severe HIE respertively. The calculeted lowest normal PI was 0.604 . PI readings below 0604 were fou nd in 2 mild, in 5 moderate and in 1 severe HIE. Even though no significative difference was found between mild and moderate HIE with regard to control group there is a clear trand of the PI to be lowest in HIE than in normal newborns.
\end{abstract}

KKey words: Uterasonic diagnosis ultrasonography, Doppler-duplex, blood flow, brain, newborn, hypoxic-ischemle encephatopathy, Pourcelot's index.

La hipoxia perinatal es una patología relativamente frecuente en nuestro medio. Su incidencia depende del adecuado manejo del embarazo de alto riesgo, de la monitorización del trabajo de parto $y$ de la eficaz seanimación del recién nacido.

La hipoxia perinatal se manifiesta por uno o mis de los siguientes factores: sufrimiento fetal agudo, Apgar bajo y acidosis metabolica ${ }^{1,2}$. Puede presentarse con variadas complicaciones sistémicas, entre las cuales se incluye la encefalopatia hipóxico-isquémica (EHI). Esta, de acuerdo a Samat y Sarnat, se clasifica en: leve, moderada y severa ${ }^{3}$. La leve se caracteriza por hiperexcitabilidad, temblores, hipertonía muscu-

1. Unidad de Radiología, Clínica Las Condes.

2. Departamento de Pediatría y Cirugía Infantil, Ciencias Médicas Occidente y Facultad de Medicina, Universidad de Chile. Unidađ de Neonatologia, Servicio de Pediatría, Hospital San Juan de Dios.

3. Departamento de Neuropsiquiatría Infantil, Hospjtal San Juan de Dios.

4. Unidad de Neonatología, Servicio de Pediatría, Hogpital San Juan de Dios. lar y llanto agudo y monótono; se recupera en 48.72 horas. La moderada incluye compromiso de conciencia tipo sopor, hipoactividad, alteración del tono muscular (cambiante de hipertonía a hipotonía) y crisis convulsivas, habitual. mente únicas, que ceden con fenobarbital; se recupera parcial o totalmente a los 7 días. La severa se caracteriza por coma profundo, hipotonía muscular generalizada, crisis convulsivas a repetición y dificultad para mantener ventilación adecuada; la mayoría fallece o queda con daño orgánico cerebral severo.

Se supone que durante la asfixia cerebral el flujo es mantenido selectivamente a las estruc. turas vitales del tronco a expensas de la corteza cerebral, la cual reaccionaría con vasoespasmo. Una vez superada la asfixia se produciria vasodilatación reactiva, lo cual aumentaría el flujo, en la mayoría de los casos, a niveles superiores a los observados antes del episodio asfíctico ${ }^{4,3}$.

El Doppler-duplex (D-D), ecografía más Doppler pulsado, ha sido usado en diversos territorios para efectuar mediciones cuantitativas o semjcuantitativas del flujo sanguineo' ${ }^{6}$. Para efec- 
tuar las mediciones semicuantitativas se han usado diversos índices que relacionan la velocidad del flujo sistólico con la velocidad del flujo diastólico. El más usado es el indice de Purce. lot (IP), que está dado por la velocidad sistólica máxima menos la velocidad diastólica mínima dividida por la velociđad sistólica máxima ${ }^{7-9}$. De este modo, una disminución de la resistencia pe. riférica se traduce en aumento del flujo diastólico, lo que se refleja, a su vez, en un descenso del IP y viceversa.

El presente trabajo contiene objetivos a corto y largo plazos. Los de corto plazo constituyen el motivo de esta publicación y son: establecer, en nuestro medio, los valores normales del IP medidos a nivel de arteria cerebral anterior, en RN de témino sanos y comparar nuestros valo. res normales con los obtenidos en un grupo de RN de término con EHI.

El objetivo a latgo plazo constituirá el motivo de una futura publicación y pretende correlacio. nar el grado de EHI y los hallazgos del D.D con el seguimiento a 2 años plazo.

\section{Pacientes y Método}

Durante dos años consecutivos (1 de marzo de 1989 al 28 de febrero de 1991) se estudiaron los RN de término con hipoxia perinatal complicados de EHI, nacidos en el Hospital San Juan de Dios. El D-D se efectuố en ba Unidad de Radjología de Clínica Las Condes. Se usó un ecógrafo Aloka 650 con unidad Doppler U.G.R. 650, con transductor mecánico de 5 Mhz. El examen se efectuó a través de fontanela anterjor, realizando inicialmente el estudio ecográfico convencional y posteriormente el estudio Doppler. Se efectuaron 6 mediciones del IP a nivel de arteria cerebral antetior, obtenicndo un promedio de ellos (figura la, b, c y d). Al alta se planificó el seguimiento neurológico.

En los 2 años estudiados hubo 49 pacientes con EHI, de los cuales 7 fallecieron en los primeros 8 días de vida, debido a la hipoxía perinatal y sus complicaciones. En 6 de los 7 niños se realiź́ estudjo anatomopatológico que confirmó el diagnóstico. En ninguno de estes 7 niños fue posible realizar el D-D cerebral. Se exchyyeron también del estudio los RN con malformaciones congénitas o que sufrieron paro cardjorrespiratotio horas después del nacimiento.

De este modo, el grupo final estudiado estuvo constitujdo por 42 niños que, de acuerdo a la clasificación de Sarnat y Sarnat, se dividieron en 22 niños con EHI leve, 16 onn EHI moderada y 4 con EHI severa.

En los nữos con EHI el peso de nacimiento promedio fue de $3410 \pm 576 \mathrm{~g}(1680-4300 \mathrm{~g})$ y su edad gestacional promedio de $39,3 \pm 1,3$ sermanas $(37 \cdot 42$ semanas). Seis correspondieron a pequeños y 7 a grandes para su edad gestacional. Treinta y tres de estos niños tenían el antecedente de stufrimiento fetal agudo intraparto. La puntuación de Apgar fue igual o menor a 5 en 35 niños al minuto, en 16 a los 5 minutos y en 6 a los 10 minutos. Además, en 30 RN se pesquisó acidosis metabólica moderada a severa en cordón umbilical o en la primera hora de vida. El D-D se efectuó en promedio a los $5,0 \pm 1,1$ días en las EHI leves, a los 6,4 = 1,8 djas en las moderadas y a los $10,8 \pm 2,2$ dias en las severas.

Paralelamente a lo anterior se efectuó el mismo estudio en un grupo de 25 RN de término sanos, adecuados para su edad gestacional. Los niños provenían también del Hospital San Juan de Dios y el estudio se efectuó entre el tercer $y$ quinto dia de vida $(\bar{x}=4,3 \pm 0,6$ días). En este grupo control el peso promedio al nacet fue de $3284 \pm 315 \mathrm{~g}(2500-3750 \mathrm{~g})$ y su edad gestacional promedio de $38,7 \pm 1,0$ semanas $(37-42$ semanas).

Para el análisis estadístico de los resultados se empleó el método de $\mathrm{t}$ Student.

\section{Resultados}

En el grupo control el IP promedio fue de $0,680 \pm 0,038$. A partir de este promedio se calculó el IP mínimo normal, el que alcanzó a 0,604 (0,680 menos 2 desviaciones estándar). En este grupo no se encontraron alteraciones ecográficas del parénquima cerebral.

En el grupo con EHI leve el IP promedio fue $0,668 \pm 0,058 ; 2$ nifios tuvieron un IP menor a $0,604(9,1 \%)$ (figura 2). No se encontraron alteraciones del parénquima cerebral ni diferencia estadísticamente significativa con el grupo control. En el grupo con EHI moderada el IP promedio fue $0,637 \pm 0,081$, encontrándose 5 niños $(31,3 \%)$ con IP menor al mínimo normal pero sin diferencia estadisticamente significativa respecto de los controles (figura 2). En 2 de es. tos niños el descenso del IP se observó al 80 y $10^{\circ}$ dia, respectivamente. De estos 5 niños, 3 presentaron además alteraciones ecográficas del parénquima cerebral (una hemorragia parietal, una lesión isquémica parietal y una lesión isquémica de los ganglios basales). En el grupo de 4 niños con EHI severa el IP promedio fue 0,677 $\pm 0,085$. Por lo pequeño del grupo no se efectuó análisis estadístico (figura 2). En 1 niño el IP se encontró disminuido al 90 día. Dos niños con IP normal presentaron alteraciones ecográficas del parénquima cerebral (una lesión isquémica focal parietal y una lesión isquémica difusa con atrofia cerebral). 

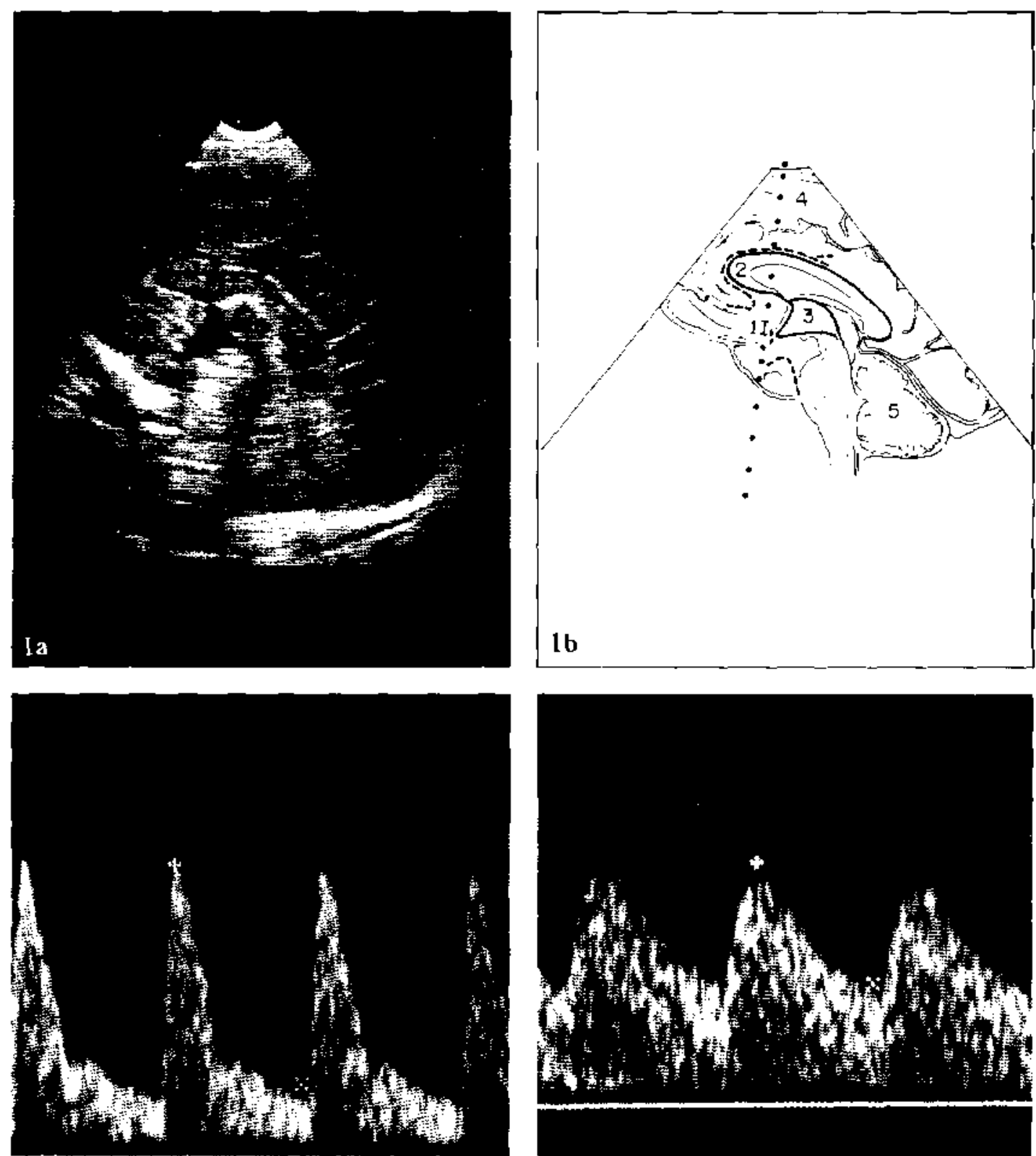

$1 \mathrm{c}$

Figura 1a: Fcografía de cerebro, corte sagital en línca media. La línea de puntos indica la dirección del Dopples y el cursor en el sitio de medición. h: Esquema de fïura la: (1) Posición del curos del Doppler en el trayecto de arteria cerebral anterior (ACA); (2) cuerpo cailoso; (3) tercer ventriculo; (4) paréncuind cerebral: (5) vérmix. c: (urva del flujo en ACA in RN de término so no con IP normal $(0,750)$. d: Curva del fluju en ACA en RN de tér. mino con EH modorada e IP bajo $(0,505)$; obsérvese el aumento dal flujo diastótico. 


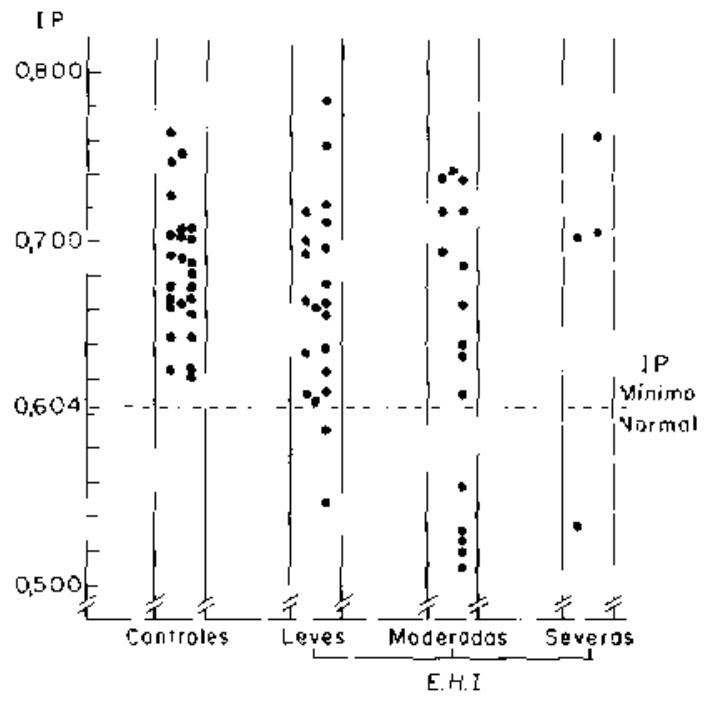

Figura 2: Distribución de los valores del IP en controles $y \mathrm{EHI}$.

\section{Discusión}

La encefalopatía hipóxico-isquémica constitu. ye una grave complicación de la hipoxia perinatal por los déficit neurológicos que puede dejar, muchos de ellos irreversibles. En el hospital de este estudio, durante 2 años consecutivos, se pesquisaron $49 \mathrm{RN}$ de término con EHI. Su incidencia alcanzó a $3,4 / 1000$ nacidos de término vivos. Esta cifra es inferior a la encontrada por Levene y cols. en Leicester, Ingiaterra, entre 1981 y $1985^{10}$.

Se sabe que en el recién nacido normal el IP disminuye desde el momento del parto hasta estabilizarse alrededor de las 48 horas de vi$\mathrm{da}^{8,21}$. Por otra parte es sabido que el $\mathbf{P}$ disminuye 24 h o más después de un episodio de asfixia y permanece bajo por varios días, habién. dose demostrado alteraciones hasta en el día $12^{\circ}$. En nuestro estudio el grupo control fue examinado, en promedio, a los 4,3 días y el grupo de EHI leve, moderada y severa fueron examinados a los $5,0,6,4$ y 10,8 días, respectivamente. Esto fue debido a que en los niños con EHl se debió esperar la mejoría de sus condicio. nes generales para poder efectuar el traslado.

El valor del IP mínimo normal encontrado por nosotros $(0,604)$ es similar a lo encontrado por Bada y cols ${ }^{7}$ y superior al descrito por otros autores ${ }^{8}, 9$. Las diferencias que expresa la literatura en el valor considerado como minimo normal son explicadas por otras tantas en los instrumentos usados y su calibración ${ }^{12}$. De aquí la importancia de que cada centro pueda contar con su propio valor mínimo nomal.

En el grupo con EHI leve, 2 nir̃os tuvieron IP disminuido. Otros ${ }^{B}$ también han encontrado disminuciones transitorias en ellas. En los casos con EHl moderada el IP promedio fue más bajo que en los controles y EHI leve; entre ellos el porcentaje de niños con IP disminuido alcanzó a 31,3\%. Esto puede tener importancia pronóstica, lo que podrá ser apreciado en el seguimiento neurológico a 2 af̂os plazo.

El hecho de que en nuestra investigación no se hayan encontrado más niños con EHI severa e IP disminuido puede ser atribuible a 3 factores: la exclusión de 7 niños con $\mathrm{EHl}$ severa que no pudieron ser estudiados, ya que fallecieron antes de ser trasladados; lo tard ro que debió ser efectuado el D-D en las EHI severas, y el pequeño tamaño de la muestra estudiada. Estos hechos explican, además, haber encontrado 3 niños (2 de ellos con alteraciones ecográficas del parénquima cerebral) con EHI seveta e IP normal, contrariamente a lo esperado, y la escasez de alteraciones ecograficas difusas del parénquima, propias del edema cerebral.

Archer y cols. en $1986^{\circ}$ encontraron que el IP deteminado entre el $3^{\circ}$ y $5^{\circ}$ día de vida, con ecógrafo portátil y al lado del RN, puede ser útil para precisar el pronóstico vital y neurológico. El D-D es un procedimiento simple, inocuo y que no prolonga el examen ecográfico convencional del cerebro por más de 5 minutos $7,13,14$. En nuestro pais, en algunos centros, esto es posible de realizar y podria ser de gran utilidad en los niños con EHI moderada y severa durante la etapa de edema cerebral y compromiso de conciencia.

En nuestra opinión el trabajo confirma que el IP puede encontrarse disminuido en las EHI. y aun cuando no se demostraron diferencias estadísticamente significativas, sí queda clara la tendencia en el sentido de que el IP disminuye en las EHI respecto de los controles.

\section{Resumen}

El Doppler-duplex (D-D) puede ser usado para cuantificar flujo sanguíneo. En cerebro pueden efectuarse mediciones semicuantitativas usando 
el indice de Pourcelot (IP). Se supone que en la etapa posthipoxia cerebral se produciría vaso. dilatación reactiva con aumento del flujo diastólico y descenso del IP. Se estudiaron $25 \mathrm{RN}$ de término sanos cuyo IP promedio fue $0,680 \pm$ 0,038 a los 4 días. En ellos el IP mínimo normal fue 0,604 . Durante 2 años consecutivos se efectuó, además, D.D en $42 \mathrm{RN}$ con EHI: 22 leves, 16 moderadas y 4 severas. En ellos el IP promedio fue $0,668 \pm 0,058,0,637 \pm 0,081$ y $0,677 \pm$ 0,085 , efectuándose el estudio a los 5,6 y 10 días, respectivamente. Dos RN con EHI leve, 5 con moderada y uno con severa iuvieron IP disminuido. Aún cuando no se demostró diferencia estadisticamente significativa entre las EHI leves y moderadas respecto del grupo control, si es clara la tendencia en cuanto a que el IP disminuye en la EHI respecto de los normales.

(Palabras claves: Ultrasonografia, Doppler, flujo cerebral, encefalopatía hipóxico-isquémica, índice de Pourcelot.)

\section{Agradecimientos}

Agradecemos a Clínica Las Condes por permitir realizar los examenes y a International Clinics (Aloka) por facilitar el trasdado de los pacientes. El apoyo constante $y$ revisión del manuscrito efectuado por el Dr. Sergio Vaisman $W_{+}$, son sinceramente reconocidos por los autores.

\section{Referencias}

1. Sykes $G$, Matloy $P$. Johnson $P$ et al Do Apgas scores indicate asphyxja? Lancet 1982; 1: 191196.

2. Gionzález $H$. Fernández $C$. Herrera $B$ : Influencia del trabajo de parto en el equilibrio ácido básico đel recién nacido. Rev Chil Pediatría 1990; 61: 91-94.

3. Sarnat $H B$, Sarnat $M S$ : Neonatal encephalopathy following fetal distress. A clinical and electroencephalographic study, Arch Neurol 1976: 33. 696705 .

4. Hossmann $X A$, Kleihues $P$ : Reversibility of ischemic brain damage. Arch Neurol 1973;29:375-384.

5. Bode $H$ : Pediatric applications of transcranial Doppler sonography. Springer-Verlag/Wien, New Yotk. 1988. pp. 49-50.

6. Burns PB: Interpretation and aralysis of Doppler signals. In Clinical application of Doppler ultrasound. Ed. Taylor KJW, Burns PN, Wells PNT, Raven Pres, New York. 1988, pp. 102-109.

7. Bada HS, Hajjar W, Chua $C$, and Stumner DS: Non invasive djagnosis of neonatal asphyxia and intraventricular hemorrage by Doppler uttrasound. J Pediatr 1979; 95 : 775-779.

8. Archer LNJ. Levene MI, Evans DH: Cercbral artery Doppler ultrasonographt for prediction of outcome after perinatal asphyxia. Lancet 1986; 2: $1116-1118$.

9. Van Bel F, Hirasing $R A$, and Grimberg is Th: Can perinatal asphyxia cause cerebral edema and affect cerebral blood flow vekcity? Eur I Pediatr 1984: 152: 29-32.

10. Levene MI, Sands $C$, Gindulis $H$, Moore $J R$ Comparison of two methods of predicting outcome in perinatal asphyxia. Lancet 1986: 1: 67-69.

11. Grov PH, Griffin EA, Drumm JE, Fitzerald DE, and Duignon NM: Continous wave Doppler ultrasound in evaluation of cerebral blood flow in neonates. Arch Dis Child 1983; 58: 677-681.

12. Volpe JJ, Perlman JM, Hill A, McMenamin JB: Cerebral blood flow velocity in the human newborn: the value of its determination. Pediatrics $1982 ; 75 \div 147-152$.

13. Drayton MR: Pediatric Doppler ultrasound. In Clinical application of Doppler ultrasound. Ed. Taylor KJW. Buts PN, Wells PNT, Raven Press, New Yotk. 1988, pp. 304-316.

14. Seibert J. McCowan TC. Chadduck WM et al.: Duplex pulsed Doppler US versus intracranial pressure in the nconate: clonical and experimental studies. Radiology 1989; 171: 155-159.

\section{AVISO A LOS AUTORES}

Con el objeto de dar prioridad a los trabajos de investigacion, en vista de las limitaciones de espacio de là Revista Chilena de Pediatría, el Comité Editorial ha acordado restringir la impresión de casos clínicos a un máximo de dos por cada número. 